\title{
ON THE RANGE OF A COERCIVE MAXIMAL MONOTONE OPERATOR IN A NONREFLEXIVE BANACH SPACE ${ }^{1}$
}

\author{
JEAN-PIERRE GOSSEZ
}

\begin{abstract}
It is shown that the range of a coercive everywhere defined maximal monotone operator from a (nonreflexive) Banach space into its dual is dense for the weak* topology but not necessarily for the norm topology.
\end{abstract}

1. Introduction. Let $X$ be a real Banach space with dual $X^{*}$, and let $T: X \rightarrow 2^{X^{*}}$ be a coercive maximal monotone operator with domain $D(T)$ and range $R(T)$. One of the basic results of monotone operator theory asserts that if $X$ is reflexive, then $R(T)$ is all of $X^{*}$ (cf. [3]). The assumption of reflexivity is essential for $T$ to be onto. However, without reflexivity, it is known that in the "selfadjoint" case, i.e. when $T$ is the subdifferential of a lower semicontinuous proper convex function, $R(T)$ is dense in $X^{*}$ for the norm topology of $X^{*}$ (this follows from the Bishop-Phelps theorem [1]). The question was raised some years ago by F. E. Browder as to whether or not this density property holds in general.

In $\S 3$ we answer this question negatively. We exhibit an everywhere defined singlevalued demicontinuous monotone operator from $l^{1}$ to $l^{\infty}$ which is coercive but whose range is not dense in $l^{\infty}$ for.the norm topology of $l^{\circ}$. On the other hand, we show in $\S 4$ that at least when $T$ is everywhere defined, a weaker density property holds, namely that $R(T)$ is weak* dense in $X^{*}$.

The construction of the above counterexample relies on a close examination of the monotone extensions of $T$ to the bidual $X^{* *}$. We remark here that the strong density property for $R(T)$ can be deduced from a suitable assumption relative to these monotone extensions (cf. [7] and \$2).

2. Monotone extensions to the bidual. Let $T: X \rightarrow 2^{X^{*}}$ be a monotone operator, and denote by $T_{1}: X^{* *} \rightarrow 2^{X^{*}}$ the operator whose graph is given

Received by the editors November 15, 1971.

AMS 1970 subject rlassifications. Primary 47H05; Secondary 46B10, 35J60.

Key words and phrase's. Nonreflexive Banach space, maximal monotone operator, density of the range, bidual space, monotone extensions, Bishop-Phelps theorem, subdifferential.

${ }^{1}$ Research supported by National Science Foundation Grant GP-28148. 
by

$$
\begin{array}{r}
\operatorname{gr} T_{1}=\left\{\left(x^{* *}, x^{*}\right) \mid \exists \text { a net }\left(x_{i}, x_{i}^{*}\right) \in \operatorname{gr} T \text { with } x_{i}\right. \text { bounded, } \\
\left.x_{i} \rightarrow x^{* *} \text { weak }^{* *} \text { and } x_{i}^{*} \rightarrow x^{*} \text { in norm }\right\} .
\end{array}
$$

( $X$ is identified with a subspace of $X^{* *}$, and cl will denote closure for the norm topology.) Clearly $T_{1}$ is a monotone extension of $T$. Under the assumption that $T_{1}$ is maximal monotone, we proved in [7] that if $T$ is coercive, then $R\left(T_{1}\right)=X^{*}$; thus, in particular, cl $R(T)=X^{*}{ }^{2}$

It might be thought that $T_{1}$ is maximal monotone as soon as $T$ is maximal monotone. Although this is the case when $T$ is a subdifferential (cf. [9], [7]) or when $T$ is the monotone operator associated with a saddlefunction (cf. [10], [8]), it is not true in general, as is seen from the following proposition.

Proposition 1. Let $A: X \rightarrow X^{*}$ be a bounded linear antisymmetric mapping such that $\left\langle x^{* *},-A^{*} x^{* *}\right\rangle$ is nonnegative for all $x^{* *} \in X^{* *}$ and positive for at least one $x^{* *} \in X^{* *}$. Then $-A^{*}$ is the unique maximal monotone extension of $A$ to the bidual, and $\operatorname{gr} A_{1} \subsetneq \operatorname{gr}\left(-A^{*}\right)$.

Proof. Clearly $-A^{*}: X^{* *} \rightarrow X^{*}$ is a maximal monotone extension of $A$. Consequently gr $A_{1} \subset \operatorname{gr}\left(-A^{*}\right)$, and since $\left\langle x^{* *}, x^{*}\right\rangle=0$ for all $\left(x^{* *}, x^{*}\right) \in$ gr $A_{1}$, we obtain $\operatorname{gr} A_{1} \subsetneq \operatorname{gr}\left(-A^{*}\right)$. To prove the uniqueness assertion, take $\left(x^{* *}, x^{*}\right)$ in the graph of a monotone extension of $A$. We have $\left\langle x^{* *}-u, x^{*}-A u\right\rangle \geqq 0$ for all $u \in X$, so that $\left\langle x^{* *}, x^{*}\right\rangle \geqq\left\langle u, x^{*}\right\rangle+\left\langle x^{* *}, A u\right\rangle$ for all $u \in X$, but this implies $x^{*}=-A^{*} x^{* *}$. Q.E.D.

EXAMPLE. Let $A: l^{1 \rightarrow} l^{x}$ be defined by $(A x)(n)=\sum_{m=1}^{\infty} x(m) K(m, n)$ for $x \in l^{1}$, where $K(m, n)=0$ if $m=n,-1$ if $n>m$ and +1 if $n<m$. Then $A$ satisfies the conditions of Proposition 1.

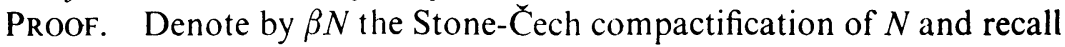
the identifications: $l^{\infty} \equiv \mathscr{C}(\beta N)$ and $\left(l^{\infty}\right)^{*} \equiv \mathscr{H}(\beta N)$. Then it is easy to ve rif that $\left\langle\mu,-A^{*} \mu\right\rangle=[\mu(\beta N \backslash N)]^{2}$ for all $\mu \in \mathscr{M}(\beta N)$, where $\mu(\beta N \backslash N)$ denotes the $\mu$-measure of $\beta N \backslash N$. Q.E.D.

We conclude this section with some remarks concerning the monotone extensions of a linear operator. Let $B: X \rightarrow X^{*}$ be a closed densely defined linear monotone mapping. Using the bipolar theorem and [7, p. 379], one obtains

$$
B_{1}=B^{* d l}
$$

where $B^{*}: X^{* *} \rightarrow X^{*}$ is the dual of $B$ and $B^{* d}: X^{* *} \rightarrow X^{*}$ the dual of $B^{*}$. Thus $D\left(B_{1}\right)=X^{* *}$ if and only if $B$ is weakly compact, and in this case,

\footnotetext{
${ }^{2}$ The proof is given in [7] under a slightly stronger assumption but it carries over
} with little change to the present situation. 
of course, $B_{1}$ is maximal monotone. Another consequence of (1), by an argument similar to [2, p. 37], is that if $B_{1}$ is maximal monotone, then $B^{*}$ is monotone. It would be of interest to know whether the converse is true. This is certainly the case when $B$ is bounded and antisymmetric, as we now briefly indicate: define the linear mapping $C: X^{* *} \rightarrow X^{*}$ by $C x^{* *}=-B^{*} x^{* *}$ for $x^{* *} \in D(C)=\left\{y^{* *} \mid\left\langle y^{* *},-B^{*} y^{* *}\right\rangle \geqq 0\right\}$; then $C$ is maximal monotone, and $C=B_{1}$ if and only if $B^{* *}(D(C)) \subset X^{*}$; but $B^{* *} x^{* *}=-B^{*} x^{* *}$ for $x^{* *} \in D(C)$.

3. Strong density property. The extension process considered in the previous section is crucial for our main result.

Proposition 2. Let $A: X \rightarrow X^{*}$ satisfy the conditions of Proposition 1 and let $J: X \rightarrow 2^{X^{*}}$ be the duality mapping. Then there exists $\lambda>0$, arbitrarily small, such that $R(\lambda J+A)$ is not dense in $X^{*}$ for the norm topology of $X^{*}$.

Taking for $A$ the mapping from $l^{1}$ to $l^{\infty}$ described in $\$ 2$ and for $J$ the duality mapping corresponding to a Gâteaux differentiable norm on $\boldsymbol{l}^{\mathbf{1}}$ (such a norm exists, cf. [5]), we obtain a coercive demicontinuous monotone operator whose range is not dense for the norm topology.

Proposition 2 is a direct consequence of the following three lemmas, where $J_{\varepsilon}: X \rightarrow 2^{X}$ is defined for $\varepsilon>0$ by

$$
J_{\varepsilon} x=\left\{x^{*} \mid\left\langle x, x^{*}\right\rangle \geqq \frac{1}{2}\|x\|^{2}+\frac{1}{2}\left\|x^{*}\right\|^{2}-\varepsilon\right\}
$$

for $x \in X$. (Note that (2) with $\varepsilon=0$ is the definition of $J$.)

LemMA 1. Let $T: X \rightarrow 2^{X^{*}}$ be a monotone operator. Let $\lambda>0$. If

$$
\operatorname{cl} R(\lambda J+T)=X^{*},
$$

then $R\left(\lambda J_{\varepsilon}+T\right)=X^{*}$ for all $\varepsilon>0$.

LEMMA 2. Let $T: X \rightarrow 2^{X^{*}}$ be a monotone operator, and suppose that for some $\varepsilon>0, R\left(\lambda J_{\varepsilon}+T\right)=X^{*}$ for all $\lambda>0$ sufficiently small. Then $R\left(T_{1}\right)=R(S)$ where $S: X^{* *} \rightarrow 2^{X^{*}}$ is any maximal monotone extension of $T$.

Lemma 3. Let $A: X \rightarrow X^{*}$ satisfy the conditions of Proposition 1. Then $-A^{*}: X^{* *} \rightarrow X^{*}$ is a maximal monotone extension of $A$, and $R\left(A_{1}\right) \subsetneq$ $R\left(-A^{*}\right)$.

Proof of Lemma 1. Take $\varepsilon>0$ and $x^{*} \in X^{*}$. We have $x^{*} \in \operatorname{cl} R(\lambda J+T)$. More precisely, using the coercivity of $\lambda J+T$, there exists $k$ such that $x^{*} \in \operatorname{cl}\{(\lambda J+T) x \mid\|x\| \leqq k\}$. But from the definition of $J_{\varepsilon}$, there exists $\eta>0$ such that $J x+B_{\eta} \subset J_{\varepsilon} x$ for all $x$ with $\|x\| \leqq k$, where $B_{\eta}$ is the ball in $X^{*}$ with center 0 and radius $\eta$. Consequently $x^{*} \in\left\{\left(\lambda J_{\varepsilon}+T\right) x \mid\|x\| \leqq k\right\}$.

Q.E.D. 
Proof of Lemma 2. Let $S: X^{* *} \rightarrow 2^{X^{*}}$ be a maximal monotone extension of $T$. Clearly gr $T_{1} \subset$ gr $S$, so that $R\left(T_{1}\right) \subset R(S)$. Now let $x^{*} \in R(S)$. By assumption, for all $\lambda>0$ sufficiently small, there exists $x_{\lambda}$ such that $x^{*}=\lambda y_{\lambda}^{*}+z_{\lambda}^{*}$ with $y_{\lambda}^{*} \in J_{\varepsilon} x_{\lambda}$ and $z_{\lambda}^{*} \in T x_{\lambda}$. We will prove that $x_{\lambda}$ remains bounded when $\lambda \downarrow 0$. It will follow, passing to a subnet if necessary, that $x_{\lambda} \rightarrow x^{* *}$ weak ${ }^{* *}$ and $z_{\lambda}^{*} \rightarrow x^{*}$ in norm (because $J_{\varepsilon}$ transforms a bounded set into a bounded set). This shows $\left(x^{* *}, x^{*}\right) \in \operatorname{gr} T_{1}$, thus $x^{*} \in R\left(T_{1}\right)$. To prove that $x_{\lambda}$ remains bounded when $\lambda \downarrow 0$, choose $u^{* *}$ with $x^{*} \in S u^{* *}$. By monotonicity $\left\langle u^{* *}-x_{\lambda}, x^{*}-z_{\lambda}^{*}\right\rangle \geqq 0$, which gives $\left\langle u^{* *}, y_{\lambda}^{*}\right\rangle \geqq\left\langle x_{\lambda}, y_{\lambda}^{*}\right\rangle$. Using the definition of $J_{\varepsilon}$, this implies $\frac{1}{2}\left\|u^{* *}\right\|^{2} \geqq \frac{1}{2}\left\|x_{\lambda}\right\|^{2}-\varepsilon$, and the conclusion follows. Q.E.D.

Proof of Lemma 3. Part of this lemma is contained in Proposition 1. It remains to see that $R\left(A_{1}\right)$ is different from $R\left(-A^{*}\right)$. We will show that if $x^{* *}$ satisfies $\left\langle x^{* *},-A^{*} x^{* *}\right\rangle>0$, then $-A^{*} x^{* *} \notin R\left(A_{1}\right)$. Suppose that $-A^{*} x^{* *}=A_{1} y^{* *}$ for some $y^{* *}$. Since $A_{1}=A^{* d}$ (cf. $\S 2$ ),

$$
\left\langle x^{* *},-A^{*} x^{* *}\right\rangle=\left\langle y^{* *}, A^{*} x^{* *}\right\rangle=\left\langle y^{* *},-A_{1} y^{* *}\right\rangle=0,
$$

a contradiction. Q.E.D.

As a corollary to Lemma 2, remark that if $T$ satisfies the assumption of Lemma 2 and admits an injective maximal monotone extension to $X^{* *}$, then $T_{1}$ is maximal monotone.

4. Weak* density property. Let $T: X \rightarrow 2^{X^{*}}$ be a monotone operator, and denote by $T_{2}: X^{* * \rightarrow \rightarrow} 2^{X^{*}}$ the operator whose graph is given by

$$
\begin{array}{r}
\operatorname{gr} T_{2}=\left\{\left(x^{* *}, x^{*}\right) \mid \exists \text { a net }\left(x_{i}, x_{i}^{*}\right) \in \operatorname{gr} T \text { with } x_{i}\right. \text { bounded, } \\
x_{i} \rightarrow x^{* *} \text { weak }^{* *}, x_{i}^{*} \rightarrow x^{*} \text { weak }^{*} \text { and } \\
\left.\lim \sup \left\langle x_{i}, x_{i}^{*}\right\rangle \leqq\left\langle x^{* *}, x^{*}\right\rangle\right\} .
\end{array}
$$

$T_{2}$ is an extension of $T$ which, in general, is larger than $T_{1}$. If $T_{1}$ is maximal monotone, then $T_{2}=T_{1}$. The definition of $T_{2}$ is closely related to the notion of an operator of type $(M)$ with respect to a subspace of its domain (cf. [4]).

Proposition 3. If $T: X \rightarrow 2^{X^{*}}$ is a coercive maximal monotone operator with $D(T)=X$, then $R\left(T_{2}\right)=X^{*}$. In particular $R(T)$ is weak dense in $X^{*}$.

Proof. The proof is based on arguments which have become standard in monotone operator theory, and we only sketch it. First note that $T$ is upper semicontinuous from the norm topology of $X$ to the weak* topology of $X^{*}$ and that for each $x \in X, T x$ is a nonempty weak* compact convex subset of $X^{*}$ (cf. [3]). It follows that if $F$ is a finite-dimensional subspace of $X$ and $i_{F}$, the injection of $F$ into $X$, the approximant operator 
$i_{F}^{*} T i_{F}: F \rightarrow 2^{F^{*}}$ has the same properties as $T$. This enables us to solve the approximant equation, and it is easy to go to the limit using the weak** compactness of the unit ball of $X^{* *}$ and the definition of $T_{2}$. Q.E.D.

Proposition 3 can be applied to the study of variational boundary value problems for quasilinear elliptic systems in divergence form with rapidly increasing coefficients. The existence results obtained in this way involve a monotonicity assumption with respect to all the derivatives of the unknown functions and are similar to those of [6]. More general existence results which only require a monotonicity assumption with respect to the top order derivatives of the unknown functions are given in the author's paper Boundary value problems for quasilinear elliptic equations with rapidly increasing coefficients, Bull. Amer. Math. Soc. (to appear).

\section{REFERENCES}

1. E. Bishop and R. R. Phelps, The support functionals of a convex set, Proc. Sympos. Pure Math., vol. 7, Amer. Math. Soc., Providence, R.I., 1963, pp. 27-35.

2. H. Brézis, On some degenerate nonlinear parabolic equations, Proc. Sympos. Pure Math., vol. 18, part 1. Amer. Math. Soc., Providence, R.I., 1970, pp. 28-38. MR 42 \#8346.

3. F. E. Browder, Nonlinear operators and nonlinear equations of evolution in Banach spaces, Proc. Sympos. Pure Math., vol. 18, part 2, Amer. Math. Soc., Providence, R.I. (to appear).

4. - Existence theory for boundary value problems for quasi-linear elliptic systems with strongly nonlinear lower order terms, Proc. Sympos. Pure Math., vol. 23, Amer. Math. Soc., Providence, R.I. (to appear).

5. M. M. Day, Strict convexity and smoothness of normed spaces, Trans. Amer. Math. Soc. 78 (1955), 516-528. MR 16, 716.

6. T. Donaldson, Nonlinear elliptic boundary value problems in Orlicz-Sobolev spaces, J. Differential Equations 10 (1971), 507-528.

7. J. P. Gossez, Opérateurs monotones non linéaires dans les espaces de Banach non réflexifs, J. Math. Anal. Appl. 34 (1971), 371-395.

8. - On the subdifferential of a saddle-function, J. Functional Analysis (to appear).

9. R. T. Rockafellar, On the maximal monotonicity of subdifferential mappings, Pacific J. Math. 33 (1970), 209-216. MR 41 \#7432.

10. - Monotone operators associated with saddle-functions and minimax problems, Proc. Sympos. Pure Math., vol. 18, part 1, Amer. Math. Soc., Providence, R.I., 1970, pp. 241-250.

Department of Mathematics, University of Chicago, Chicago. Illinois 60637

Department of Mathematics, University of Brussels, Brussels, Belgium (Current address) 